\section{Is there value in a two-step diagnostic algorithm to confirm SARS-CoV-2 in South Africa?}

To the Editor: SARS-CoV-2 molecular platforms that attained Food and Drug Administration Emergency Use Authorizations are currently being implemented worldwide. ${ }^{[1-4]}$ These molecular platforms incorporate at least two gene targets, with positive percent agreements (PPA) of $95-100 \%$ and negative percent agreements (NPA) of $94-100 \% \cdot{ }^{[1-4]}$ The South African (SA) Ministerial Advisory Group on COVID-19 recently outlined a mass screening programme involving a broadened case definition, active community surveillance and extensive contact tracing, with the aim of undertaking $~ 30000$ molecular SARS-CoV-2 screening tests daily in SA. ${ }^{[5]}$

We performed a hypothetical predictive study, using an average test prevalence of 3\% (based on SA confirmed COVID-19-positive cases/ total cases tested up to 22 April 2020) ${ }^{[6]}$ and test PPA and NPA of $99 \%$, taking into consideration possible pre-analytical and analytical confounders. ${ }^{[7]}$ The positive predictive value (PPV) and negative predictive value (NPV) for SA were $75.4 \%$ and $99.9 \%$, respectively. We also compared predictive values, using similar accuracy, of other countries that have implemented different testing and screening strategies (based on figures on 22 April 2020) ${ }^{[8,9]}$ (Table 1).
Traditionally, for disease screening, a high NPV and sensitivity are ideal. ${ }^{[10]}$ Given the current situation in SA, reporting false-positive SARS-CoV-2 cases may be justified based on facilitating social measures to contain the spread of the virus. ${ }^{[10]}$ However, when disease prevalence is low, such as the current SA COVID-19 test prevalence of $3 \%$, near-perfect specificity would be necessary to prevent false positives. ${ }^{[7]}$ The prevention of false positives is particularly important when extensive contact tracing is instituted, where the appropriate use of resources is essential. ${ }^{[1,12]}$ Other possible issues are legal implications due to infringement of freedom rights and loss of personal income due to self-isolation. ${ }^{[13]}$

Several solutions may decrease false positives when the test prevalence remains low, including:

- Performing reflex confirmatory molecular tests, using separate platforms with different targets, on screening positive samples. In our scenario, secondary confirmatory testing of positive samples, using a platform with similar accuracy, would increase the PPV to 99.7\% (Table 1). However, confirmatory testing may delay contact tracing, depending on local testing capacity. Combined highthroughput screening and low-throughput confirmatory platforms may mitigate this. A cost-and-risk analysis comparing a single-test screening strategy and a two-step algorithm could be undertaken.

Table 1. A hypothetical two-step model to confirm SARS-CoV-2 in South Africa and comparison with other countries ${ }^{\star}$

\begin{tabular}{|c|c|c|c|}
\hline & True positive & True negative & Total \\
\hline \multicolumn{4}{|c|}{ South Africa: test prevalence 3\% } \\
\hline \multicolumn{4}{|l|}{ Screening, $n^{\dagger}$} \\
\hline Test positive & 3599 & 1301 & 4900 \\
\hline Test negative & 36 & 128838 & 128874 \\
\hline Total & 3635 & 130139 & 133774 \\
\hline \multicolumn{4}{|l|}{ Confirmatory, $n^{\ddagger}$} \\
\hline Test positive & 3658 & 12 & 3670 \\
\hline Test negative & 37 & 1193 & 1230 \\
\hline Total & 3695 & 1205 & 4900 \\
\hline \multicolumn{4}{|c|}{ South Korea: test prevalence $2 \%$} \\
\hline \multicolumn{4}{|l|}{ Screening, $n^{\S}$} \\
\hline Test positive & 10587 & 5281 & 15868 \\
\hline Test negative & 107 & 522800 & 522907 \\
\hline Total & 10694 & 528081 & 538775 \\
\hline \multicolumn{4}{|c|}{ Italy: test prevalence $12 \%$} \\
\hline \multicolumn{4}{|l|}{ Screening, $n^{5}$} \\
\hline Test positive & 185454 & 13227 & 198681 \\
\hline Test negative & 1873 & 1309446 & 1311319 \\
\hline Total & 187327 & 1322673 & 1510000 \\
\hline \multicolumn{4}{|c|}{ USA: test prevalence $22 \%$} \\
\hline \multicolumn{4}{|l|}{ Screening, $n^{1}$} \\
\hline Test positive & 840230 & 30213 & 870443 \\
\hline Test negative & 8487 & 2991070 & 2999557 \\
\hline Total & 848717 & 3021283 & 3870000 \\
\hline \multicolumn{4}{|c|}{ UK: test prevalence $24 \%$} \\
\hline \multicolumn{4}{|l|}{ Screening, $n^{\star *}$} \\
\hline Test positive & 132160 & 4264 & 136424 \\
\hline Test negative & 1335 & 422176 & 423511 \\
\hline Total & 133495 & 426440 & 559935 \\
\hline \multicolumn{4}{|c|}{ 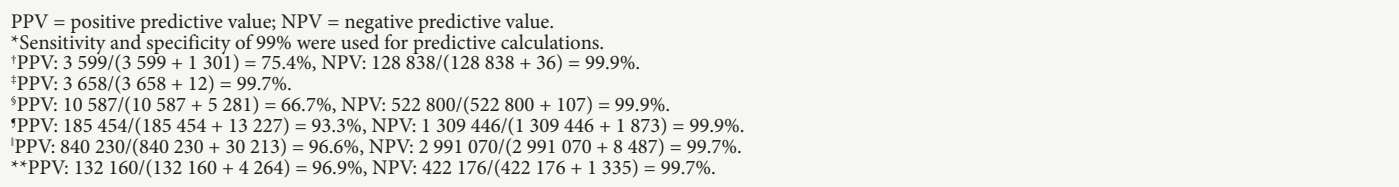 } \\
\hline
\end{tabular}


- Implementation of risk stratification based on disease grading, and only doing confirmatory tests on 'low'-risk cases (i.e. lower pre-test probability), may be more cost-effective but would be administratively challenging.

- Narrowing the case definition would improve the pre-test probability, but risks missing COVID-19 cases and minimises the benefits of the contact tracing programme.

We fully support the SA National Department of Health $(\mathrm{NDoH})$ in expanding testing and contact tracing, and this letter is not intended as a criticism of the $\mathrm{NDoH}$ response, which has been widely praised. Our intention is to initiate discussion around the acknowledged challenges of mass screening and tracing programmes, and thereby, we hope, contribute in some way to the collective efforts in combating COVID19 in SA. Consistent low test prevalence of SARS-CoV-2 might rationalise a two-step diagnostic algorithm to support cost-effective mass contact testing and tracing. A reference test standard remains lacking, and NPA values of current testing modalities highlight the need for further COVID-19 diagnostic accuracy studies. ${ }^{[1,2,4]}$

Acknowledgements. We thank Profs A Whitelaw and A Brink, heads of the divisions of medical microbiology at the universities of Stellenbosch and Cape Town, respectively, for their input and review of this letter.

\section{W Dowling}

Division of Medical Microbiology, National Health Laboratory Service and Faculty of Medicine and Health Sciences, Stellenbosch University, Cape Town, South Africa

wentzel.dowling@nhls.ac.za

\section{J Opperman}

Division of Medical Microbiology, Faculty of Medicine and Health Sciences, Stellenbosch University, Cape Town, South Africa; and National Health Laboratory Service, Tygerberg Hospital, Cape Town, South Africa

1. Food and Drug Administration. Xpert Xpress SARS-CoV-2: For use with GeneXpert Dx or GeneXpert Infinity Systems. April 2020. https://www.fda.gov/media/136314/download (accessed 16 April 2020).

2. Cheng MP, Papenburg J, Desjardins M, et al. Diagnostic testing for severe acute respiratory syndromerelated coronavirus-2. Ann Intern Med 2020 (epub 13 April 2010). https://doi.org/10.7326/m20-1301

3. Poljak M, Korva M, Knap Gašper N, et al. Clinical evaluation of the cobas SARS-CoV-2 test and a Poljak M, Korva M, Knap Gašper N, et al. Clinical evaluation of the cobas SARS-CoV-2 test and a
diagnostic platform switch during 48 hours in the midst of the COVID-19 pandemic. J Clin Microbiol 2020 (epub 10 April 2020). https://doi.org/ $/ 10.1128 / \mathrm{jcm} .00599-20$
diagnostic plattorm switch during 48 hours in the midst of the

4. Seegene Inc. Summary of Allplex ${ }^{\mathrm{m}} 2019$-nCoV Assay performance data. http://www.seegene.com/ assays/allplex_2019_ncov_assay\# (accessed 18 April 2020).

5. National Department of Health, South Africa. SA's COVID-19 epidemic: Trends \& next steps. 13 April 2020. https://sacoronavirus.co.za/2020/04/13/sas-covid-19-epidemic-trends-next-steps/ (accessed 17 April 2020)

6. National Institute for Communicable Diseases. COVID-19 update. 20 April 2020. https://www.nicd. ac.za/covid-19-update-36/ (accessed 17 April 2020).

7. Van Zyl G, Maritz J, Newman H, Preiser W. Lessons in diagnostic virology: Expected and unexpected sources of error. Rev Med Virol 2019;29(4):e2050. https://doi.org/10.1002/rmv.2052

8. Worldometer. Coronavirus cases. https://www.worldometers.info/coronavirus/coronavirus-cases/ \#daily-cases (accessed 23 April 2020).

9. Our World in Data. COVID-19 testing dataset. To understand the global pandemic, we need global testing. https://ourworldindata.org/covid-testing (accessed 23 April 2020).

10. Bedford J, Enria D, Giesecke J, et al. COVID-19: Towards controlling of a pandemic. Lancet 2020;395(10229):1015-1018. https://doi.org/10.1016/s0140-6736(20)30673-5 11. Hellewell J, Abbott S, Gimma A, et al. Feasibility of controlling COVID-19 outbreaks by isolation
of cases and contacts. Lancet Glob Health 2020;8(4):e488-e496. https://doi.org/10.1016/s2214of cases and con
$109 \mathrm{x}(20) 30074-7$

12. Colquhoun D. An investigation of the false discovery rate and the misinterpretation of $p$-values. R Soc Open Sci 2014;1(3):140216. https://doi.org/10.1098/rsos.140216

13. Adams S, Lindeque G, Soma-Pillay P. Bioethics and self-isolation: What about low-resource settings? S Afr Med J 2020;110(5):350-352. https://doi.org/10.7196/SAMJ.2020.v110i5.14733

S Afr Med J 2020;110(7):564-565. https://doi.org/10.7196/SAMJ.2020.v110i7.14853 